\title{
Smart Parking Application
}

\author{
Tejal Lotlikar \\ Computer Engineering \\ Department \\ Vivekanand Education \\ Society's Institute of \\ Technology \\ Mumbai, India
}

\author{
Minla Chandrahasan \\ Computer Engineering \\ Department \\ Vivekanand Education \\ Society's Institute of \\ Technology \\ Mumbai, India
}

\author{
Ankita Mahadik \\ Computer Engineering \\ Department \\ Vivekanand Education \\ Society's Institute of \\ Technology \\ Mumbai, India
}

\author{
Madhusmita Oke \\ Computer Engineering Department \\ Vivekanand Education Society's Institute of \\ Technology \\ Mumbai, India
}

\author{
Anjali Yeole \\ Computer Engineering Department \\ Vivekanand Education Society's Institute of \\ Technology \\ Mumbai, India
}

\begin{abstract}
The ever increasing population has led to chaotic city traffic As a result of the process of searching a parking lot becomes tedious. It is time consuming task leading to discomfort. The fuel consumption is on an increasing side due to such scenarios. The increase in vehicular traffic creates a negative impact on the environment. In the wake of smart city times these issues lead us to the need of a 'smart' solution. In order to resolve these issues and satisfy the increasing demand for the parking areas, parking management organizations are trying to implement better and technologically advanced solutions. A cloud based smart parking application will enable real time parking availability monitoring and reservation thereby providing better services to the end users as well as reduce the workload of the parking administrator.
\end{abstract}

\section{General Terms}

Your general terms must be any term which can be used for general classification of the submitted material such as Pattern Recognition, Security, Algorithms et. al.

\section{Keywords}

Keywords are your own designated keywords which can be used for easy location of the manuscript using any search engines.

\section{INTRODUCTION}

\subsection{Motivation}

The increase in city traffic is one of the major effects of population growth especially in urban areas. Due to this searching for a vacant parking area during peak hours is not only time-consuming but also results in wastage of fuel. The drivers keep searching for suitable parking lot which leads to increase in traffic. Increasing volume of vehicular exhaust creates a negative impact on the environment. Hence reservation-based smart parking has become the need of the day.

\subsection{Problem Definition}

The Smart Parking Application aims at helping users to find the most suitable area for parking, make reservations and extend them, if required. It enables parking administrators to define and manage parking spaces as well as enables parking operators to authenticate users against their reservations when users enter the parking area. Users access location based information and request system services via mobile applications and parking operators verify reservations via mobile applications whereas parking admins may manage the parking area details via a web application. The smart parking application features use of cloud computing to improve user services.

\subsection{Relevance of project}

In order to satisfy the increasing demand for the parking areas, parking management organizations are trying to implement better and technologically advanced solutions. A variety of methodologies have been implemented abroad to disseminate parking availability information through various platforms. The smart parking application will enable real-time parking availability checking and reservation thereby providing a hassle-free parking solution for users. Deployment over the cloud not only frees administrators from maintenance tasks but also makes the system more environment friendly.

\section{RELATED WORK}

Current systems proposed for smart parking include

\subsection{Smart parking using RFID [1]}

RFID technology is an automation technology with main components as RFID readers, labels, computers, barriers, software etc. The software is for management, controlling, transaction reporting and operation tasks for parking lots. This vehicular data is fetched from database to verify a vehicle when it enters the parking lot using RFID reader. When a vehicle wants to check out, the authentication is done by analysing check-in details. The drivers will be notified about the availability of parking lots at the entrance itself. Hence, no time is wasted looking for park space.

\subsection{Reservation based smart-parking [2]}

Reservation based smart parking system aims at broadcasting real time parking information to drivers via an application. The system mainly uses Zigbee sensors and smart phone support for deployment. This system enables the users to reserve a particular parking space in advance by accessing its availability information in specific parking district. It is also equipped with payment option including dynamic pricing for users. Light and vibration sensors detect whether a vehicle is present in the lot. Bluetooth module is responsible for communication between user and the sensor information. The major disadvantages include shorter range of bluetooth and bottleneck situations may arise in case of heavy traffic. 


\subsection{Smart parking reservation system using [3]}

This system enables users to reserve their parking places using SMS. Once the reservation is confirmed, the users will receive the one time password(OTP) to enter the parking area and the lot number for parking. If they exceed the time to reach the parking area, the password will expire and the reservation will be cancelled. The system is subdivided into Reservation and Access system. The reservation is handled by a micro-RTU (Remote Terminal Unit) configured with SSE OPC (OLE for Process Control) Server. The access system is handled by a microcontroller-IC16F877- which measures time periods for validity of passwords, stores/provides information on the availability of parking spaces as well as allowing or denying access to the parking area. A weight sensor is positioned at the lot area to sense the presence of the vehicle.

\subsection{ParkNet : Drive by sensing road-side parking statistics[4]}

ParkNet is a mobile system comprising of vehicles that collect parking space occupancy information by driving by. The vehicle includes a GPS receiver and passenger-side facing ultrasonic rangefinder to determine vacant parking spaces. The data collected is integrated at the central server producing a real-time map of parking availability thereby serving user's requests. In order to achieve accuracy of location, environmental fingerprinting approach is devised. It makes use of GPS module and ultrasonic sensors for end to end communication. A real-time map is generated to reflect the occupancy with respect to a timestamp. The drawbacks are mainly due to limitations of sensors and complexity involved in cases of multi-lane parking.

\section{REQUIREMENTS}

\subsection{Functional Requirements}

Our proposed system comprises of a web application for parking area admins, a mobile application for parking operators and a mobile application for users.

Each of their requirements are as stated below.

\subsubsection{Web Application for parking admin}

- Define new parking areas, specify number of parking lots, the parking cost per minute/hour and other details.

- Modify data of existing parking areas.

- View the data of all registered parking areas.

\subsubsection{Mobile Application for parking operator}

- Send vehicle plate number and reservation password (Session ID) to central server for verification when users check in.

- Issue bill to users on checkout.

\subsubsection{Mobile Application for end users}

- Register for the service and enter personal and vehicle details.

- $\quad$ Find a parking area from the list of areas, registered by parking admins.

- View the details of a selected parking area such as the name, price per minute, number of total available lots.
- Reserve an available parking lot and specify duration of reservation.

\subsubsection{Backend Management System}

- Authenticate users and admins before modifying any sensitive data

- Accept reservation of parking lots based on availability

- Generate a Session ID for each reservation and send it to the user

- Allow modification of parking lot status by operators

- Auto-cancel reservation if user fails to reach within the window period.

\subsection{Non-functional Requirements}

- The central repository should be platform independent so that it can be accessible and store application data via the web application and the mobile app.

- The server should be able to handle concurrent requests from different users.

- The system should provide confidentiality for user data using database encryption and local encryption to protect data in the event of device theft (laptop/handheld device).

- The android application provides high availability and high accuracy in finding the locations.

\subsection{Constraints}

\subsubsection{Stable internet connection}

The proposed system requires a stable internet connection for efficient functioning. In case of internet disconnection, the system may fail. The user, parking operators and parking administrator interact with the system using mobile application and web application via internet. Hence stable internet connection is the basic requirement of this system.

\subsubsection{Highly Scalable Architecture}

The proposed smart parking system should be highly scalable. Using cloud services for database provides scalability as the as the entire database can be stored in the cloud and can be scaled as per need.

\subsubsection{Handling concurrent access to the centralized database}

The smart parking system should be able to handle concurrent access to the database by users. As the database is centralized, many users can simultaneously access it and it is possible that many users can book the same parking area at the same time. The system must be able to handle such cases.

\subsubsection{Availability}

As the user is using the smart parking service through a mobile application, real time availability status should be delivered to the user as and when required i.e. the system should be easily available to end users.

\subsection{Hardware and Software Requirements}

The proposed systems can be implemented using a suitable cloud computing platform such as Amazon Web Services (AWS) or Microsoft Azure. The requirements for deployment using AWS are as stated below. 


\subsubsection{Hardware Requirements}

3.4.1.1 Web Application

- Web server : AWS Elastic Compute Cloud (EC2)

- Database : MySQL database

3.4.1.2 Mobile Application

- Backend server : AWS Elastic Compute Cloud (EC2)

- Database: MySQL database

\subsubsection{Software Requirements}

3.4.2.1 Web Application

- XAMPP

\subsubsection{Mobile Application}

- $\quad$ Android Studio

3.4.2.3 Database Management

- Remote Desktop Connection

\section{PROPOSED DESIGN}

\subsection{System Architecture}

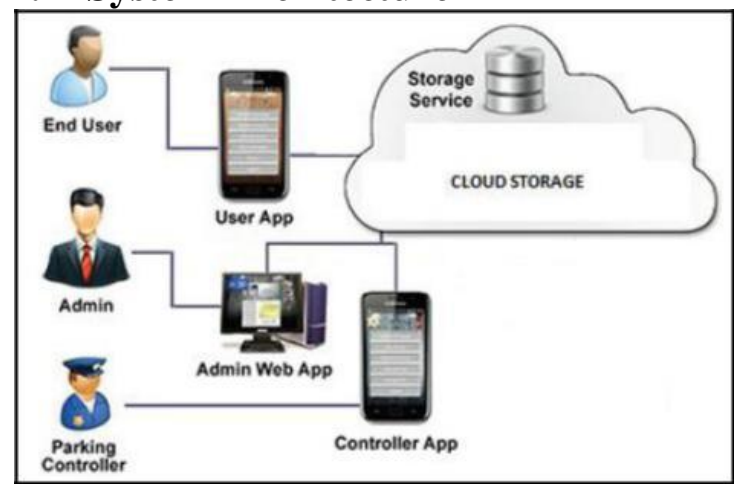

\subsection{Conceptual System Design}

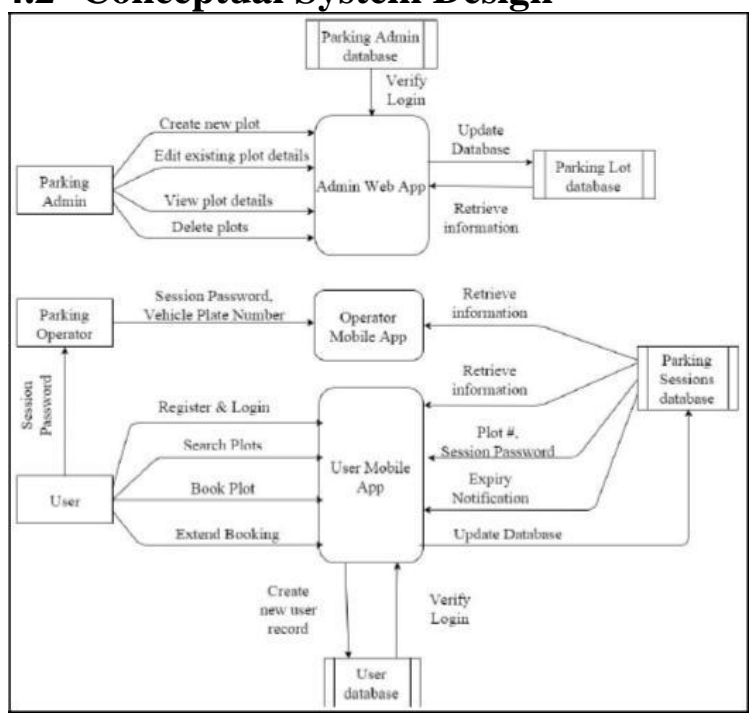

\section{IMPLEMENTATION}

The smart parking application is collectively implemented in following modules:

- Website for the Administrator

- Mobile Application for the End-user

- Mobile Application for the Parking Operator

A single database stores information regarding parking areas, users and their reservations.

The database consists of tables: admin, operator, parkingarea, parkinglots, parkingsession, user, user_cars

\subsection{Website for Administrator}

Website enables Administrator to perform functions: Add, View and Edit a parking area.
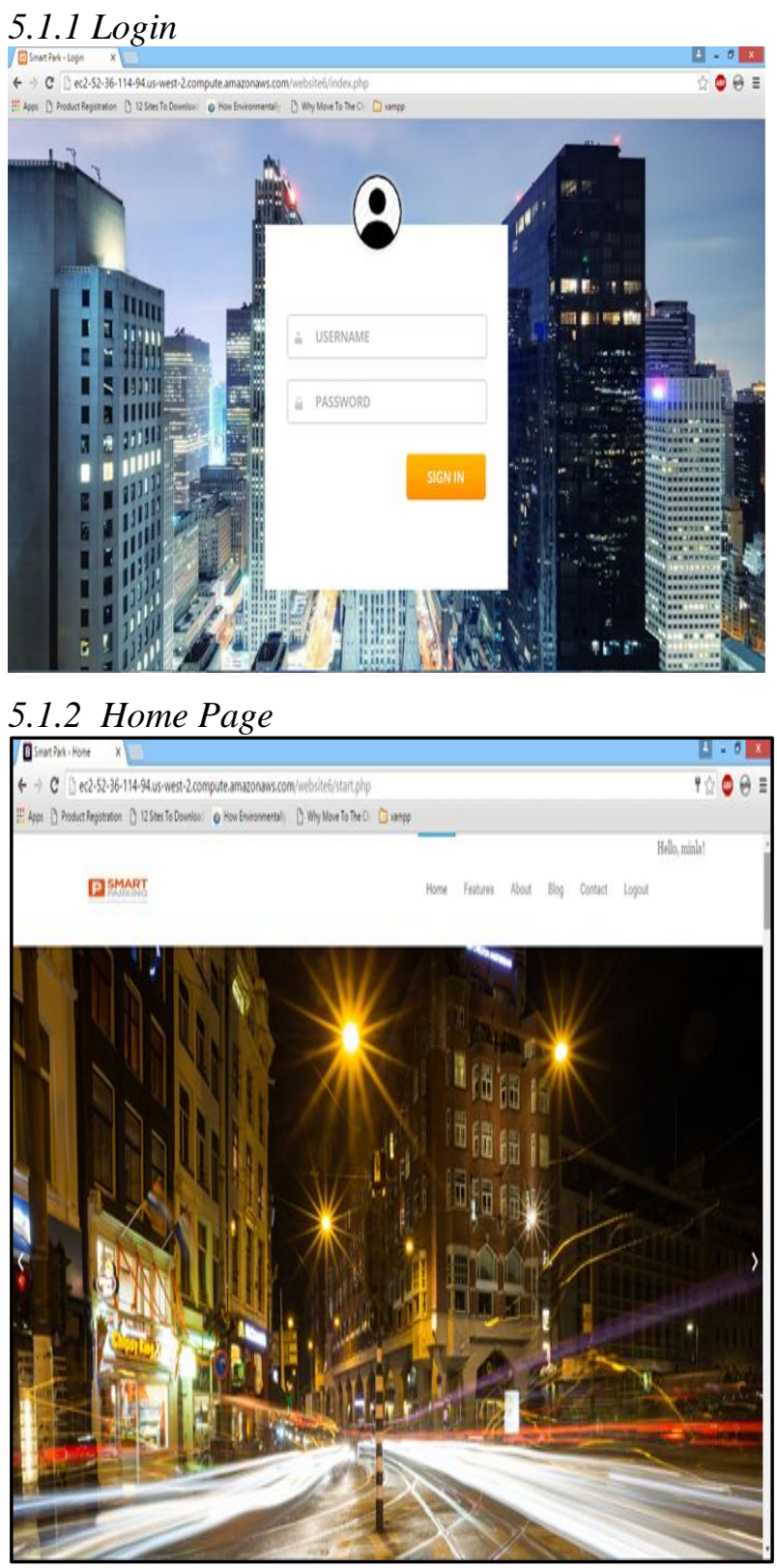


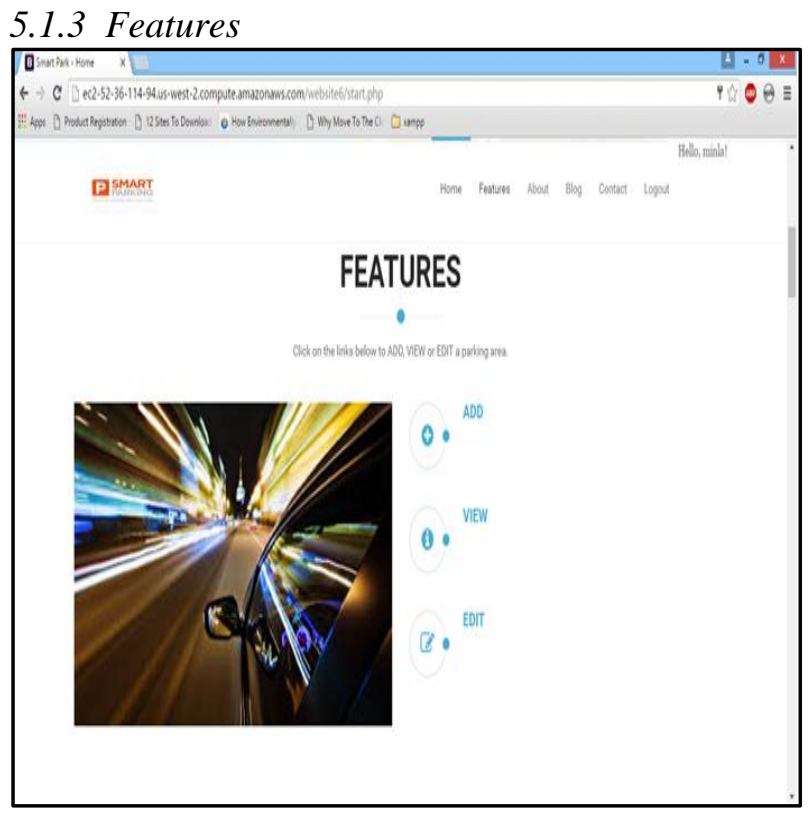

5.1.3.1 Adding a new parking area

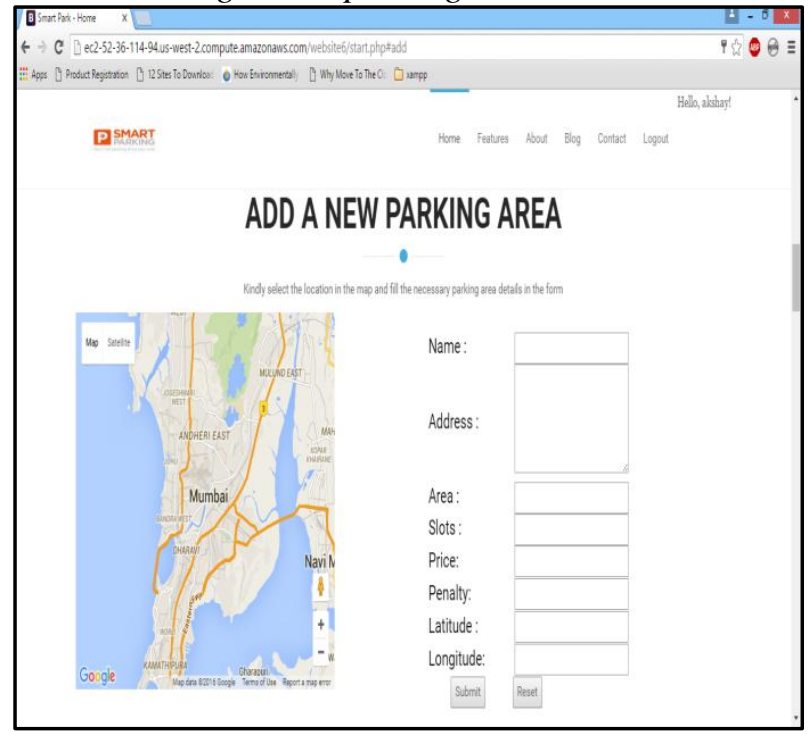

5.1.3.2 View parking area details

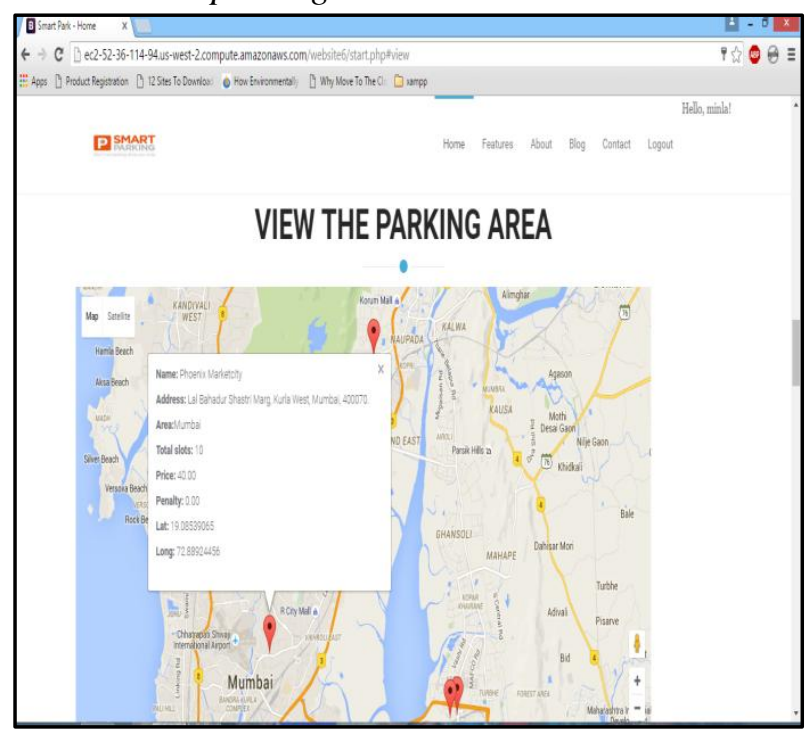

\subsubsection{Edit parking area details}

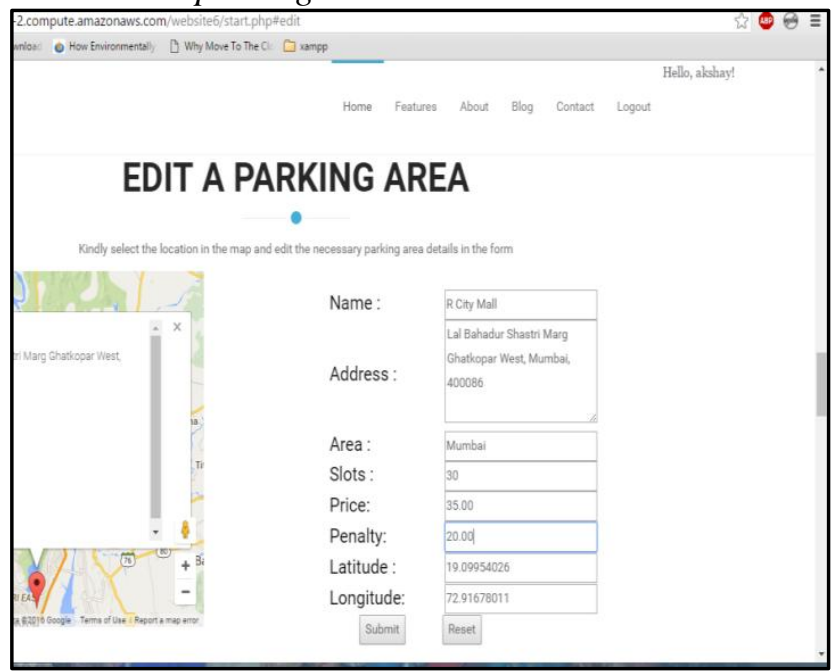

\subsection{Mobile Application for Operator}

Mobile Application for the Operator helps in Authentication and Billing functions.

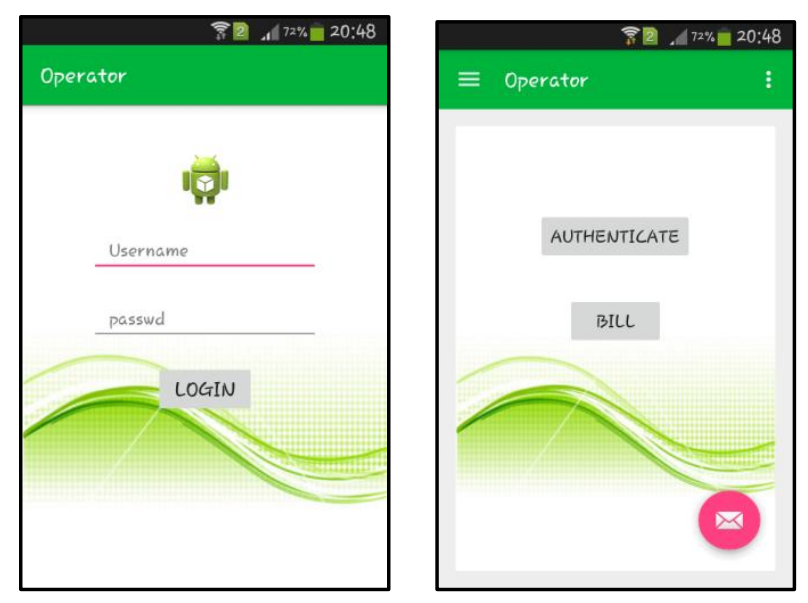

5.3 Mobile Application for End User

Mobile Application for the End-user provides functions Search, Book, View, Cancel slots.

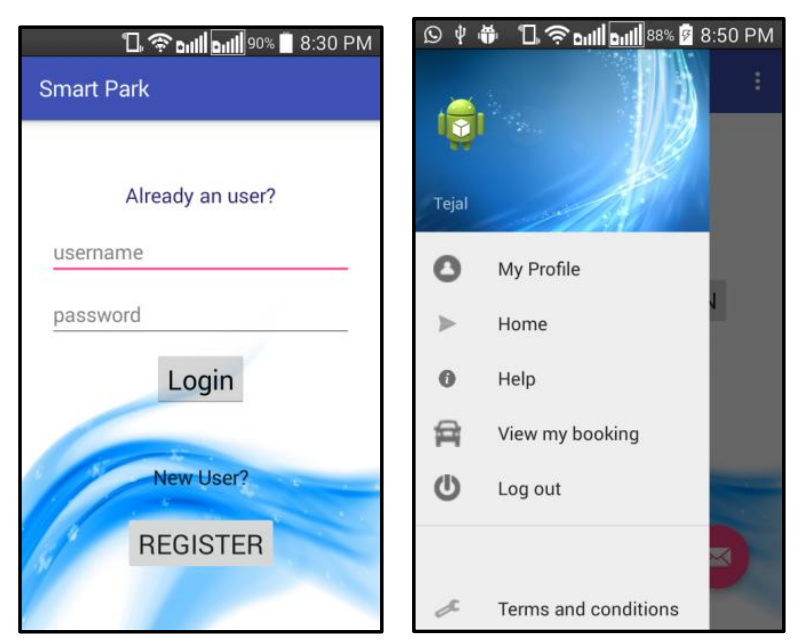




\section{DRAWBACKS OF EXISTING SYSTEMS}

Drawbacks found in existing systems are:

\subsection{RFID System}

This system is considerably expensive. The RFID tags are application specific, no one tag fits all, more than one tag can respond at the same time. This system fails in case of internet disconnection. If two vehicles enter a parking lot side by side, being within the range of the RFID reader, the system will not read their identification information and process it. Electromagnetic spectrum signals are relatively easy to jam using energy at the right frequency.

\subsection{Reservation based Smart Parking System}

The reservation based smart parking system is expensive and also difficult to maintain. The problem of bottleneck may occur due to heavy traffic.

\subsection{Smart Parking Reservation System Using SMS}

The system is a standalone system i.e. The system is designed for individual use by parking areas, and not a centralized system encompassing reservation for all parking areas within a region. As the system uses CMOS flash memory for storage of parking information, the database can be scaled within hardware limitations.

\subsection{PARKNET Drive by sensing roadside statistics}

Errors in providing accuracy of locations and occupancy. It is expensive and more complex due to lane detection mechanism. If the vehicle does not move within a specified speed range there may occur failure of ultrasonic sensors to detect distances.

\section{RESULTS AND DISCUSSIONS \\ 7.1 Administrator functions}

\subsubsection{Add Slot:}

On selecting an area on the map, its latitude and longitude is obtained by the system and then the admin has to fill in the remaining details.

\subsubsection{View slot:}

On selecting a marker on the map, the admin can view the details of that parking area.

\subsection{Edit Parking Area Details:}

The admin can edit the parking area details by editing the form displayed after viewing the slot

\subsection{Operator functions}

\subsubsection{Authentication:}

When user enters parking area, operator verifies reservation using session id and car number of the user. Before Authentication (when the user arrives at the parking area), the status of parking lot is 'booked' which is later changed to 'occupied' when the user enters the parking area after authentication.

\subsubsection{Billing:}

The operator enters the car no and session id to get the bill generated for a particular through the mobile spplication. On billing, out-time registered, bill amount calculated and updated, status changed to 'finished' and Parking lot status changed to 'available' and total number of parking lots in the given parking area are incremented.

\subsection{End-user functions:}

\subsubsection{Search parking area:}

On selecting a location, user can view detailed list of parking areas, their availability and the pricing.

\subsubsection{Book Parking lot:}

The user is required to enter id of the required parking area and duration of reservation to book a lot as per availability. After booking, a unique session id is given to user to confirm booking.

\subsubsection{View booking:}

The user can view his current booking details like location, session id and status of booking through the end user's application.

\subsubsection{Cancel booking:}

The user can also can his current booking. On cancelling, parking session status becomes "cancelled" and availability of slots gets automatically updated.

\section{ADVANTAGES}

In comparison to the existing systems available for smart parking, the system proposed by us makes use of cloud computing because of which it is:

\subsection{Cost effective}

Sensors and other cost intensive hardware are not employed. No infrastructure maintenance involved. You simply have to pay as per use.

\subsection{Environment Friendly}

The cloud uses energy in a more streamlined and efficient way than traditional, in-house data centers. Cloud computing uses multi-tenant architecture and this tends to be more efficient than the typical, single-tenant, statically-allocated data centers.

\subsection{Highly available}

The system will be available to the end users provided they have an internet connection and the smart parking mobile application

\subsection{Easy to use}

Users can access the parking details and parking operators perform verifications using a user friendly mobile application. The admin can also perform respective functions using a web application without hassle of infrastructure maintenance.

\subsection{Highly scalable}

The entire database stored in cloud can be scaled as per need.

\subsection{Robust}

The system is highly robust as cloud providers offer comprehensive backup and recovery capabilities across various services and platforms.

\section{CONCLUSION}

Due to advancement in technology, drivers are demanding easier and less time-consuming parking facilities. There are various methodologies of smart parking that have been implemented to provided better services to the end users and improve the overall management of the existing parking system. The real time monitoring of available parking lots and allotment of the suitable parking area by advanced reservation 
are some of the characteristic services provided to the customers or end users through a user friendly interface like a mobile application or using SMS services.

After analyzing the different solutions, it is observed that there are some drawbacks of each system like excessive use of expensive sensor modules, difficulty in sensing accurate parking availability data due to speed constraints of the vehicle, use of certain modules like Bluetooth that function efficiently only for short distance, inefficient user authentication techniques and centralized management of the database of parking areas in different regions. These drawbacks need to be eliminated to enhance the overall performance of the system.

\section{REFERENCES}

[1] Z. Pala and N. Inanc, "Smart parking applications using RFID technology" in $1^{\text {st }}$ Annual Eurasia RFID conference, September 2007.

[2] Wand and W. He, "A reservation based smart parking system" in $1^{\text {st }}$ Int.'l Workshop on Cyber-Physical networking systems, April 2011.

[3] N.H.H.M. Hanif, M.H. Badiozaman and H. Daud, "Smart parking reservation system using short message services (SMS)", in 2010 International Conference on Intelligent and Advanced Systems (ICIAS), June 2010.

[4] Brabham, "Crowdsourcing as a model for problem solving: An introduction and Cases" Convergence: The International Journal of Research into New Media Technlogical Studies.

[5] ].S. Mathur, T. Jin, N. Kasturirangan, W. Xue, M. Gruteser and W. Trappe, "Parknet : drive by sensing of road-side parking statistics" in Proceedings of the Eighth International Conference on Mobile Systems, applications and services (MobiSys'10), ACM New York, June 2010.

[6] Elena Polycarpou, Lambros Lambrinos and Eftychios Protopapadakis, "Smart Paking Solutions for Urban Areas"

[7] Saeed Arbabi, Mohammad Allahbakhsh, Mohsen Sharifi, "Crowd-Enhanced Cloud Services: Issues and Directions", International Journal of Computer Applications (0975 - 8887) Volume 117 - No. 21, May 2015 